Series A

\author{
I. MATHEMATICA
}

435

\title{
ON BOUNDED UNIVALENT FUNCTIONS WHICH ARE CLOSE TO IDENTITY
}

BY

MENAHEM SCHIFFER and OLLI TAMMI

H E L S I N K I 1968

S U O M A L A I N E T I E D E A K A T E M I A

doi:10.5186/aasfm.1969.435 
Communicated 11 October 1968 by Olli Lehto and K. I. Virtanen 


\section{Introduction}

The role of the Grunsky inequalities for the coefficient problem in the theory of univalent functions is well known. Using the inequalities, one could prove the Bieberbach conjecture for functions which are close enough to the Koebe function [2]. The purpose of the present paper is to give a similar treatment to the coefficient problem for bounded univalent functions which are close enough to the identity mapping $f(z)=z$. The Grunsky inequalities have been sharpened for the case of bounded univalent functions by Nehari [4]. Recently we have extended the result of Nehari [5] and this allows us to give a precise solution of the coefficient problem in this case.

In order to lay the groundwork and to exemplify our method, we deal in Section 1 with the special case of $a_{7}$. Here all the features of the general case are already present, while we can give a specific condition for proximity to the identity in order that our result be valid. In Section 2 we prepare some formal identities and asymptotic expressions needed in the general treatment. In Section 3 we consider the case of odd indexed coefficients $a_{2 n+1}$ under the assumption that $a_{2}, \ldots, a_{n-1}$ vanish. This special case is particularly easy to handle and prepares the more complicated approach to the general case. In Section 4, finally, the case of arbitrary $a_{2 n-1}$ is settled. Now we proceed to deal with even indexed coefficients $a_{2 n}$. This is achieved in Sections $5-7$ by introducing the odd function $\sqrt{f\left(z^{2}\right)}$ and applying the previous results.

\section{The case of $a_{7}$}

Let $S\left(b_{1}\right)$ denote the family of analytic functions

$$
f(z)=\sum_{\nu=1}^{\infty} b_{\nu} z^{\nu}=b_{1} \sum_{\nu=1}^{\infty} a_{\nu} z^{\nu}, \quad a_{\nu}=\frac{b_{v}}{b_{1}},
$$

which are univalent in $|z|<1$ and bounded, that is,

$$
|f(z)|<1, \quad 0<b_{1} \leqq 1 .
$$

Our aim is to give an estimate for the coefficients $a_{v}$ when $b_{1}$ is close to 1 .

This work was supported in part by contract AF 49 (638) 1345 at Stanford University. 
Our tools in the investigation are the following concepts and inequalities. Let

$$
\log \frac{f(z)-f(\zeta)}{z-\zeta}=\sum_{i, k=0}^{\infty} A_{i k} z^{i} \zeta^{k}
$$

and

$$
-\log \left(1-f(z) \overline{f(\zeta))}=\sum_{i, k=1}^{\infty} B_{i k} z^{i} \bar{\zeta}^{k} .\right.
$$

The matrices $\left(\left(A_{i k}\right)\right)$ and $\left(\left(B_{i k}\right)\right)$ are symmetric and hermitean, respectively. We recently showed the following necessary and sufficient condition for $f(z)$ to be univalent and bounded in $|z|<1$ : The inequality

$$
\operatorname{Re}\left\{\sum_{m, n=0}^{N} A_{m n} x_{m} x_{n}\right\}+\sum_{m, n=1}^{N} B_{m n} x_{m} \bar{x}_{n} \leqq \sum_{m=1}^{N} \frac{\left|x_{m}\right|^{2}}{m}(N=1,2, \ldots)
$$

must hold for every complex vector $\left\{x_{n}\right\}$. The case $x_{0}=0$ is the Nehari condition for univalent bounded functions; it appears, however, that the introduction of the additional variable $x_{0}$ is very important in the discussion of the coefficient problem.

In the case $N=3$, an easy calculation leads to the relations

$$
\begin{aligned}
A_{00}= & \log b_{1} \\
A_{11}= & a_{3}-a_{2}^{2} \\
A_{22}= & a_{5}-\frac{3}{2} a_{3}^{2}-2 a_{2} a_{4}+4 a_{2}^{2} a_{3}-\frac{3}{2} a_{2}^{4}, \\
A_{33}= & a_{7}-2 a_{4}^{2}-3 a_{3} a_{5}-2 a_{2} a_{6}+4 a_{2}^{2} a_{5}+12 a_{2} a_{3} a_{4} \\
& +\frac{7}{3} a_{3}^{3}-15 a_{2}^{2} a_{3}^{2}-8 a_{2}^{3} a_{4}+14 a_{2}^{4} a_{3}-\frac{10}{3} a_{2}^{6} \\
A_{01}= & a_{2}, \\
A_{02}= & a_{3}-\frac{1}{2} a_{2}^{2}, \\
A_{03}= & a_{4}-a_{2} a_{3}+\frac{1}{3} a_{2}^{3}, \\
A_{12}= & a_{4}-2 a_{2} a_{3}+a_{2}^{3} \\
A_{13}= & a_{5}-a_{3}^{2}-2 a_{2} a_{4}+3 a_{2}^{2} a_{3}-a_{2}^{4}, \\
A_{23}= & a_{6}-2 a_{2} a_{5}-3 a_{3} a_{4}+5 a_{2} a_{3}^{2}+4 a_{2}^{2} a_{4}-7 a_{2}^{3} a_{3}+2 a_{2}^{5} ;
\end{aligned}
$$




$$
\begin{aligned}
& B_{11}=b_{1}^{2}, \\
& B_{22}=\frac{1}{2} b_{1}^{4}+\left|b_{2}\right|^{2}, \\
& B_{33}=\frac{1}{3} b_{1}^{6}+2 b_{1}^{2}\left|b_{2}\right|^{2}+\left|b_{3}\right|^{2}, \\
& B_{12}=b_{1} \bar{b}_{2}, \\
& B_{23}=b_{1}^{3} \bar{b}_{2}+b_{2} \bar{b}_{3}, \\
& B_{13}=b_{1} \bar{b}_{3} .
\end{aligned}
$$

In order to estimate $a_{7}$, take $x_{3}=1$. The coefficients $a_{6}$ and $a_{5}$ are eliminated by choosing

$$
\left\{\begin{array}{l}
x_{2}=a_{2} \\
x_{1}=\frac{1}{2}\left(3 a_{3}-a_{2}^{2}\right) .
\end{array}\right.
$$

From [4] we know that the best possible estimate for $a_{7}$ is obtained for

$$
x_{0}=\frac{\operatorname{Re}\left\{\sum_{1}^{3} A_{0 u} x_{u}\right\}}{\log b_{1}^{-1}}=\frac{\operatorname{Re}\left\{a_{4}+\frac{3}{2} a_{2} a_{3}-\frac{2}{3} a_{2}^{3}\right\}}{\log b_{1}^{-1}} .
$$

Thus, for $N=3$, (5) assumes the form

$$
\begin{gathered}
a_{7}-\frac{1}{3}\left(1-b_{1}^{6}\right) \\
\leqq \\
\quad \operatorname{Re}\left\{2 a_{4}^{2}+a_{2}^{3} a_{4}-3 a_{2} a_{3} a_{4}-\frac{19}{12} a_{3}^{3}+\frac{25}{4} a_{2}^{2} a_{3}^{2}\right. \\
\left.+\frac{19}{4} a_{2}^{4} a_{3}+\frac{13}{12} a_{2}^{6}-\frac{1}{2}\left(3+5 b_{1}^{2}\right) a_{3} \bar{a}_{2}^{2}\right\} \\
-\frac{1}{4}\left(9-25 b_{1}^{2}\right)\left|a_{3}\right|^{2}+\frac{1}{4}\left(1-b_{1}^{2}\right)\left|a_{2}\right|^{4}+\frac{1}{2}\left(1-9 b_{1}^{4}\right)\left|a_{2}\right|^{2} \\
\left.\operatorname{Re}\left\{a_{4}+\frac{3}{2} a_{2} a_{3}-\frac{2}{3} a_{2}^{3}\right\}\right]^{2} .
\end{gathered}
$$


We rewrite this condition by using the notation

$$
\begin{gathered}
t=a_{4}+\frac{3}{2} a_{2} a_{3}-\frac{2}{3} a_{2}^{3} ; \\
a_{7}-\frac{1}{3}\left(1-b_{1}^{6}\right) \leqq 2 \operatorname{Re}\left\{t^{2}\right\}-\frac{[\operatorname{Re}\{t\}]^{2}}{\log b_{1}^{-1}} \\
+\operatorname{Re}\left\{-9 a_{2} a_{3} t-\frac{19}{12} a_{3}^{3}+\frac{11}{3} a_{2}^{3} t+\frac{61}{4} a_{2}^{2} a_{3}^{2}-\frac{17}{2} a_{2}^{4} a_{3}+\frac{95}{36} a_{2}^{6}\right. \\
\left.-\frac{1}{2}\left(3+5 b_{1}^{2}\right) \bar{a}_{2}^{2} a_{3}\right\}+\frac{1}{4}\left(1-b_{1}^{2}\right)\left|a_{2}\right|^{4} \\
\div \frac{1}{4}\left(9-25 b_{1}^{2}\right)\left|a_{3}\right|^{2}+\frac{1}{2}\left(1-9 b_{1}^{4}\right)\left|a_{2}\right|^{2} .
\end{gathered}
$$

In order to estimate the right side of (10), we will utilize the following bounds ([5], [6]):

$$
\begin{aligned}
& \left|a_{2}\right| \leqq 2\left(1-b_{1}\right), \text { for all } b_{1} ; \\
& \left|a_{3}\right| \leqq 1-b_{1}^{2} \leqq 2\left(1-b_{1}\right), \text { for } e^{-1} \leqq b_{1} \leqq 1 ; \\
& \left|a_{4}\right| \leqq \frac{2}{3}\left(1-b_{1}^{3}\right) \leqq 2\left(1-b_{1}\right), \text { for } 0,65 \leqq b_{1} \leqq 1 ; \\
& \left|a_{\nu}\right| \leqq 2\left(1-b_{1}\right), \text { for } e^{-1} \leqq b_{1} \leqq 1, v=2,3,4 .
\end{aligned}
$$

This gives for $t$

$$
|t| \leqq\left[2+6\left(1-b_{1}\right)+\frac{16}{3}\left(1-b_{1}\right)^{2}\right]\left(1-b_{1}\right) .
$$

When restricting ourselves to

$$
0,9 \leqq b_{1} \leqq 1
$$

we obtain $|t|<2,7\left(1-b_{1}\right)$. Thus, for the interval (11) the following inequalities are valid:

$$
\left\{\begin{array}{l}
\mid a_{v} \vdots \leqq 2\left(1-b_{1}\right), \quad v=2,3,4 ; \\
|t|<2,7\left(1-b_{1}\right) .
\end{array}\right.
$$

In view of (11), the first part of the right side of (10) may be estimated as follows:

$$
2 \operatorname{Re}\left\{t^{2}\right\}-\frac{[\operatorname{Re}\{t\}]^{2}}{\log b_{1}^{-1}} \leqq[\operatorname{Re}\{t\}]^{2}\left(2-\frac{1}{\log b_{1}^{-1}}\right) \leqq 0 .
$$


Hence, in the interval considered,

$$
\begin{aligned}
& a_{7}-\frac{1}{3}\left(1-b_{1}^{6}\right) \leqq \frac{1}{4}\left(9-25 b_{1}^{2}\right)\left|a_{3}\right|^{2}+\frac{1}{2}\left(1-9 b_{1}^{4}\right)\left|a_{2}\right|^{2} \\
& +\operatorname{Re}\left\{-9 a_{2} a_{3} t-\frac{19}{12} a_{3}^{3}-\frac{1}{2}\left(3+5 b_{1}^{2}\right) \bar{a}_{2}^{2} a_{3}+\frac{11}{3} a_{2}^{3} t+\frac{61}{4} a_{2}^{2} a_{3}^{2}\right. \\
& \left.-\frac{17}{2} a_{2}^{4} a_{3}+\frac{1}{4}\left(1-b_{1}^{2}\right)\left|a_{2}\right|^{4}+\frac{95}{36} a_{2}^{6}\right\}=\mathrm{I}+\mathrm{II} .
\end{aligned}
$$

Here we have divided the right side into the parts

$$
\begin{aligned}
\mathrm{I}= & \frac{4}{5} \cdot \frac{1}{4}\left(9-25 b_{1}^{2}\right)\left|a_{3}\right|^{2}+\frac{2}{3} \cdot \frac{1}{2}\left(1-9 b_{1}^{4}\right)\left|a_{2}\right|^{2}+\operatorname{Re}\left\{-9 a_{2} a_{3} t\right\}, \\
\mathrm{II}= & \frac{1}{5} \cdot \frac{1}{4}\left(9-25 b_{1}^{2}\right)\left|a_{3}\right|^{2}+\frac{1}{3} \cdot \frac{1}{2}\left(1-9 b_{1}^{4}\right)\left|a_{2}\right|^{2} \\
& +\operatorname{Re}\left\{-\frac{19}{12} a_{3}^{3}-\frac{1}{2}\left(3+5 b_{1}^{2}\right) \bar{a}_{2}^{2} a_{3}+\frac{11}{3} a_{2}^{3} t+\frac{61}{4} a_{2}^{2} a_{3}^{2}\right. \\
& \left.-\frac{17}{2} a_{2}^{4} a_{3}+\frac{1}{4}\left(1-b_{1}^{2}\right)\left|a_{2}\right|^{4}+\frac{95}{36} a_{2}^{6}\right\} .
\end{aligned}
$$

For I we get in view of (12)

$$
\mathrm{I} \leqq \frac{1}{5}\left(9-25 b_{1}^{2}\right)\left|a_{3}\right|^{2}+\frac{1}{3}\left(1-9 b_{1}^{4}\right)\left|a_{2}\right|^{2}+25\left(1-b_{1}\right)\left|a_{2}\right|\left|a_{3}\right| .
$$

The discriminant on the right side of the above expression, considered as a quadratic form in $\left|a_{2}\right|$ and $\left|a_{3}\right|$, is given by

$$
\frac{1}{5}\left(9-25 b_{1}^{2}\right) \cdot \frac{1}{3}\left(1-9 b_{1}^{4}\right)-\left(\frac{25}{2}\right)^{2}\left(1-b_{1}\right)^{2} \text {. }
$$

This is positive in the interval defined by (11). Hence, in that interval $\mathrm{I} \leqq 0$.

Next, we decompose $\mathrm{II}=\mathrm{II}_{1}+\mathrm{II}_{2}$ :

$$
\begin{aligned}
\mathrm{II}_{1} & =\frac{1}{20}\left(9-25 b_{1}^{2}\right)\left|a_{3}\right|^{2}+\operatorname{Re}\left\{-\frac{19}{12} a_{3}^{3}\right\}, \\
\mathrm{II}_{2} & =\frac{1}{6}\left(1-9 b_{1}^{4}\right)\left|a_{2}\right|^{2}+\operatorname{Re}\left\{-\frac{1}{2}\left(3+5 b_{1}^{2}\right) \bar{a}_{2}^{2} a_{3}\right. \\
& \left.+\frac{11}{3} a_{2}^{3} t+\frac{61}{4} a_{2}^{2} a_{3}^{2}-\frac{17}{12} a_{2}^{4} a_{3}+\frac{1}{4}\left(1-b_{1}^{2}\right)\left|a_{2}\right|^{4}+\frac{95}{36} a_{2}^{6}\right\} .
\end{aligned}
$$




$$
\mathrm{II}_{1} \leqq\left|a_{3}\right|^{2}\left[\frac{1}{20}\left(9-25 b_{1}^{2}\right)+\frac{19}{6}\left(1-b_{1}\right)\right]
$$

Here []$<0$ for the interval (11).

$$
\begin{aligned}
\mathrm{II}_{2} & \leqq\left|a_{2}\right|^{2}\left[\frac{1}{6}\left(1-9 b_{1}^{4}\right)+\left(3+5 b_{1}^{2}\right)\left(1-b_{1}\right)\right. \\
& +\frac{11}{3} \cdot 2 \cdot 2,7 \cdot\left(1-b_{1}\right)^{2}+61\left(1-b_{1}\right)^{2} \\
& \left.+\frac{17}{3} \cdot 2\left(1-b_{1}\right)^{3}+2\left(1-b_{1}\right)^{3}+\frac{95}{9} \cdot 4\left(1-b_{1}\right)^{4}\right] .
\end{aligned}
$$

The factor [ ] is seen to be $<0$ for

$$
0,93 \leqq b_{1} \leqq 1
$$

Hence we proved the

$$
\text { Theorem: } 0 \leqq a_{7} \leqq \frac{1}{3}\left(1-b_{1}^{6}\right) \text { at least for } 0,93 \leqq b_{1} \leqq 1 \text {. }
$$

\section{The structure of the coefficients $A_{i k}$ and $B_{i k}$}

We shall discuss the asymptotic character of the coefficients $A_{i k}$ and $B_{i k}$ in the series developments (3) and (4) in the case that the first coefficient $b_{1}$ is close to 1 . This will allow us to utilize the inequalities (5) to estimate the coeffcients $a_{j}$ of the function $f(z) \in S\left(b_{1}\right)$ considered. To clarify the situation, we set up

$$
f(z)=b_{1}\left(z+\sum_{\nu=2}^{n} a_{\nu} z^{\nu}+\sum_{\nu=n+1}^{\infty} a_{\nu} z^{\nu}\right)
$$

It will appear that in the asymptotics for $A_{i k}$ and $B_{i k}$ with $i \leq n$, $k \leq n$ the first set of coefficients plays a different role than the second set. Indeed, we obtain in view of (3) the identity

$$
\begin{aligned}
\sum_{i, k=0}^{\infty} A_{i k} z^{i} \zeta^{k}= & \log b_{1}+\log \left[1+\sum_{\nu=2}^{n} \sum_{\alpha=0}^{v-1} a_{\nu} z^{\alpha} \zeta^{\nu-\alpha-1}\right. \\
& \left.+\sum_{\nu=n+1}^{\infty} \sum_{\alpha=0}^{\nu-1} a_{\nu} z^{\alpha} \zeta^{\nu-\alpha-1}\right] .
\end{aligned}
$$

If we develop the right-hand side into a formal power series in $z$ and $\zeta$ and wish to express in this way all $A_{i k}$ with $i, k \leq n$, we may disregard all terms which are of degree of homogeneity $>2 n$. We thus find 
(16)

$$
\begin{aligned}
& \sum_{i, k=0}^{\infty} A_{i k} z^{i} \zeta^{k}=\log b_{1}+\sum_{\nu=2}^{n} \sum_{\alpha=0}^{\nu-1} a_{v} z^{\alpha} \zeta^{\nu-\alpha-1} \\
& +\sum_{\nu=n+1}^{\infty} \sum_{\alpha=0}^{\nu-1} a_{\nu} z^{\alpha} \zeta^{\nu-\alpha-1}-\frac{1}{2}\left[\left(\sum_{\nu=2}^{n} \sum_{\alpha=0}^{\nu-1} a_{\nu} z^{\alpha} \zeta^{\nu-\alpha-1}\right)^{2}\right. \\
& +2 \sum_{\nu=2}^{n} \sum_{\alpha=0}^{\nu-1} a_{\nu} z^{\alpha} \zeta^{\nu-\alpha-1} \cdot \sum_{\nu=n+1}^{\infty} \sum_{\alpha=0}^{\nu-1} a_{\nu} z^{\alpha} \zeta^{\nu-\alpha-1} \\
& \left.+a_{n+1}^{2}\left(\sum_{\alpha=1}^{n} z^{\alpha} \zeta^{n-\alpha}\right)^{2}\right]+D
\end{aligned}
$$

where $D$ is small of the third order in all $a_{k}$ and is of second order at least in $a_{2}, \ldots, a_{n}$ and does not contain terms of degree $\leqq 2 n$. Comparing now the coefficients of $z^{i} \zeta^{k}$ on both sides of (14), we find

$$
\begin{gathered}
A_{i k}=a_{i+k+1}-\sum_{v=2}^{i} v a_{v} a_{i+k+2-v} \\
-\frac{1}{2} \sum_{v=i+1}^{k+1}(i+1) a_{\nu} a_{i+k+2-v}+\ldots
\end{gathered}
$$

for $i \leqq k=0, \ldots, n$. If $a_{v}$ with $v<2$ occurs in these formulas, it is understood to be zero.

In particular,

$$
A_{n n}=a_{2 n+1}-\sum_{\nu=2}^{n} v a_{v} a_{2 n+2-v}-\frac{n+1}{2} a_{n+1}^{2}+\ldots .
$$

The omitted terms are of third order in all $a_{k}$ and quadratic in $a_{2}, \ldots, a_{n}$.

Similarly, we consider the generating function for the matrix $\left(\left(B_{i k}\right)\right)$. We have

$$
\sum_{i, k=1}^{\infty} B_{i k} z^{i} \bar{\zeta}^{k}=-\log (1-f(z) \overline{f(\zeta)})
$$

and in view of the representation (14) for $f(z)$ we find

$$
\begin{aligned}
& \sum_{i, k=1}^{\infty} B_{i k} z^{i} \bar{\zeta}^{k}=-\log \left(1-b_{1}^{2} z \bar{\zeta}\right) \\
& -\log \left(1-b_{1}^{2} \frac{z \sum_{2}^{\infty} \bar{a}_{\nu} \bar{\zeta}^{\nu}+\bar{\zeta} \sum_{2}^{\infty} a_{\nu} z^{\nu}+\sum_{2}^{\infty} a_{v} z^{\nu} \sum_{2}^{\infty} \bar{a}_{\nu} \bar{\zeta}^{v}}{1-b_{1}^{2} z \bar{\zeta}}\right)
\end{aligned}
$$

Clearly, we find 
(20)

$$
\begin{gathered}
B_{i k}=b_{1}^{2 i} \frac{1}{i} \delta_{i k}+\left[b_{1}^{2} \frac{z \sum_{2}^{n} \bar{a}_{\nu} \bar{\zeta}^{\nu}+\bar{\zeta} \sum_{2}^{n} a_{v} z^{v}+\sum_{2}^{n} a_{v} z^{\nu} \sum_{2}^{n} \bar{a}_{\nu} \bar{\zeta}^{v}}{1-b_{1}^{2} z \bar{\zeta}}\right]_{i, k} \\
+\frac{1}{2}\left[\left(b_{1}^{2} \frac{z \sum_{2}^{n} \bar{a}_{v} \bar{\zeta}^{\nu}+\bar{\zeta} \sum_{2}^{n} a_{\nu} z^{\nu}}{1-b_{1}^{2} z \bar{\zeta}}\right)^{2}\right]_{i, k}+\ldots
\end{gathered}
$$

for $i, k \leqq n$. Here $[\Phi(z, \zeta)]_{i, k}$ denotes the coefficient of $z^{i} \zeta^{k}$ in the development $\Phi(z, \zeta)$. The terms not written down are at least cubic in $a_{2}, \ldots, a_{n}$, that is,

$$
\begin{aligned}
B_{i k} & =\frac{1}{i} b_{1}^{2 i} \delta_{i k}+a_{i-k+1} b_{1}^{2 k}+\bar{a}_{k-i+1} b_{1}^{2 i} \\
& +\sum_{\mu=2}^{k} a_{\mu+i-k} \bar{a}_{\mu} b_{1}^{2(k-\mu+1)}+\frac{1}{2} \sum_{\alpha=1}^{i-1} \sum_{\beta=1}^{k-1}\left[a_{\alpha-\beta+1} b_{1}^{2 \beta}\right. \\
& \left.+\bar{a}_{\beta-\alpha+1} b_{1}^{2 \alpha}\right]\left[a_{i-k-(\alpha-\beta)+1} b_{1}^{2(k-\beta)}+\bar{a}_{k-i-(\beta-\alpha)+1} b_{1}^{2(i-\alpha)}\right]+\ldots
\end{aligned}
$$

As before, the deleted terms are at least of third order in all $a_{k}$ and quadratic in $a_{2}, \ldots, a_{n}$. Again, $a_{1}$ is defined to be zero.

Let us display in particular the term

$$
\begin{aligned}
B_{n n} & =\frac{1}{n} b_{1}^{2 n}+\sum_{\mu=2}^{n}\left|a_{\mu}\right|^{2} b_{1}^{2(n-\mu+1)} \\
& +\frac{1}{2} \sum_{\alpha=1}^{n-1} \sum_{\beta=1}^{n-1}\left[a_{\alpha-\beta+1} b_{1}^{2 \beta}+\bar{a}_{\beta-\alpha+1} b_{1}^{2 \alpha}\right] \\
& \cdot\left[a_{-(\alpha-\beta)+1} b_{1}^{2(n-\beta)}+\bar{a}_{-(\beta-\alpha)+1} b_{1}^{2(n-\alpha)}\right]+\ldots
\end{aligned}
$$

Clearly, $a_{\varrho}$ is non-zero only for $\varrho \geqq 2$. Hence, the last double sum reduces to

$$
\sum_{\alpha \geqq \beta+1}\left|a_{\alpha-\beta+1}\right|^{2} b_{1}^{2(\beta+n-\alpha)}
$$

and we arrive at the result

$$
\begin{aligned}
B_{n n} & =\frac{1}{n} b_{1}^{2 n}+\sum_{\mu=2}^{n}\left|a_{\mu}\right|^{2} b_{1}^{2(n-\mu+1)} \\
& +\sum_{\mu=2}^{n}(n-\mu)\left|a_{\mu}\right|^{2} b_{1}^{2(n-\mu+1)}+\ldots .
\end{aligned}
$$




\section{The case $a_{2}=\ldots=a_{n}=0$.}

We apply the above formulas to solve the following:

Problem. The coefficient $a_{2 n+1}>0$ is to be estimated under the side conditions

$$
a_{2}=\ldots=a_{n}=0 .
$$

The coefficients $a_{n+1}, \ldots, a_{2 n}$ are free parameters of the problem.

Choose in the Nehari-inequalities (5) $N=n, x_{n}=1$ and

$$
x_{0}=\frac{\operatorname{Re}\left\{\sum_{1}^{n} A_{0 \mu} x_{\mu}\right\}}{\log b_{1}^{-1},},
$$

which is the most favorable choice for $x_{0}$. We thus get

$$
\begin{aligned}
& \operatorname{Re}\left\{\sum_{i, k=1}^{n} A_{i k} x_{i} x_{k}\right\}+\sum_{i, k=1}^{n} B_{i k} x_{i} \bar{x}_{k} \\
& \leqq-\frac{\left[\operatorname{Re}\left\{\sum_{k=1}^{n} A_{0 k} x_{k}\right\}\right]^{2}}{\log b_{1}^{-1}}+\sum_{1}^{n} \frac{\left|x_{i}\right|^{2}}{i} .
\end{aligned}
$$

In the case (24) in question, the higher order corrections in (17), (21) and (23) vanish. Thus,

$$
\begin{aligned}
& \left\{\begin{aligned}
A_{i k} & =a_{i+k+1} ; 0 \leqq i \leqq k \leqq n, i<n, \\
A_{n n} & =a_{2 n+1}-\frac{n+1}{2} a_{n+1}^{2} ;
\end{aligned}\right. \\
& \left\{\begin{aligned}
B_{k k} & =\frac{1}{k} b_{1}^{2 k} \\
k & =1, \ldots, n
\end{aligned}\right. \\
& \left\{\begin{aligned}
B_{i k} & =0 \\
1 & \leqq
\end{aligned}\right.
\end{aligned}
$$

In view of (24), the inequality (25) assumes the form 


$$
\begin{aligned}
& \operatorname{Re}\left\{x_{1} \sum_{k=n-1}^{n} a_{k+2} x_{k}+x_{2} \sum_{k=n-2}^{n} a_{k+3}+\ldots\right. \\
& +x_{q} \sum_{k=n-q}^{n} a_{k+q+1} x_{k}+\ldots+x_{n-1} \sum_{k=1}^{n} a_{k+n} x_{k} \\
& \left.+x_{n}\left(\sum_{k=1}^{n-1} a_{k+n+1} x_{k}+a_{2 n+1}-\frac{n+1}{2} a_{n+1}^{2}\right)\right\} \\
& +\sum_{k=1}^{n} \frac{1}{k} b_{1}^{2 k}\left|x^{k}\right|^{2} \\
& \leqq-\frac{\left[\operatorname{Re}\left\{a_{n+1}\right\}\right]^{2}}{\log b_{1}^{-1}}+\sum_{k=1}^{n} \frac{\left|x_{k}\right|^{2}}{k}
\end{aligned}
$$

that is,

$$
\text { (26) } \quad \begin{aligned}
& a_{2 n+1}-\frac{1}{n}\left(1-b_{1}^{2 n}\right) \\
& \leqq \\
& \quad\left(\frac{n+1}{2}-\frac{1}{\log b_{1}^{-1}}\right)\left[\operatorname{Re}\left\{a_{n+1}\right\}\right]^{2}-\frac{n+1}{2}\left[\operatorname{Im}\left\{a_{n+1}\right\}\right]^{2} \\
& -\operatorname{Re}\left\{x_{1} \sum_{n-1}^{n} a_{k+2} x_{k}+x_{2} \sum_{n-2}^{n} a_{k+3} x_{k}+\ldots\right. \\
& +x_{q} \sum_{n-q}^{n} a_{k+q+1} x_{k}+\ldots+x_{n-1} \sum_{1}^{n} a_{k+n} x_{k} \\
& \left.+\sum_{1}^{n-1} a_{k+n+1} x_{k}\right\}+\sum_{1}^{n-1} \frac{1}{k}\left(1-b_{1}^{2 k}\right)\left|x_{k}\right|^{2} .
\end{aligned}
$$

We will study the possible maximum of the left side by choosing the parameters $x_{1}, \ldots, x_{n-1}$ properly. The result is obtained step by step as follows.

$$
1^{\circ} x_{1}=\ldots=x_{n-1}=0
$$

$$
a_{2 n+1}-\frac{1}{n}\left(1-b_{1}^{2 n}\right)
$$

$$
\leqq\left(\frac{n+1}{2}-\frac{1}{\log b_{1}^{-1}}\right)\left[\operatorname{Re}\left\{a_{n+1}\right\}\right]^{2}-\frac{n+1}{2}\left[\operatorname{Im}\left\{a_{n+1}\right\}\right]^{2}
$$

Here $\frac{n+1}{2}-\frac{1}{\log b_{1}^{-1}} \leqq 0$ for

$$
e^{-\frac{2}{n+1}} \leqq b_{1}<1
$$


Equality occurs only in the case $b_{1}=e^{-\frac{2}{n+1}}$. Consider the general case where $\frac{n+1}{2}-\frac{1}{\log b_{1}^{-1}}<0$, which means that

$$
e^{-\frac{2}{n+1}}<b_{1}<1 \text {. }
$$

The left-hand side in (27) is non-positive and zero only if

$$
a_{n+1}=0 .
$$

We continue to discuss the case of equality in the estimate for $a_{2 n+1}-\frac{1}{n}\left(1-b_{1}^{2 n}\right)$. In the interval (28) the condition (26) can be utilized by putting $a_{n+1}=0$ on its right side.

$$
2^{\circ} \quad x_{2}=\ldots=x_{n-1}=0
$$

The inequality (26) assumes the form

$$
a_{2 n+1}-\frac{1}{n}\left(1-b_{1}^{2 n}\right) \leqq-2 \operatorname{Re}\left\{x_{1} a_{n+2}\right\}+\left(1-b_{1}^{2}\right)\left|x_{1}\right|^{2} .
$$

In case $x_{1}=\bar{a}_{n+2}$, we have the estimate

$$
a_{2 n+1}-\frac{1}{n}\left(1-b_{1}^{2 n}\right) \leqq-\left|a_{n+2}\right|^{2}\left(1+b_{1}^{2}\right) .
$$

Thus, the equality in this estimate requires that necessarily

$$
a_{n+2}=0 .
$$

This process can be repeated without restriction $n$ times, and we end up at the following result:

Theorem. Suppose that $a_{2}=\ldots=a_{n}=0$ and let $a_{n+1}, \ldots, a_{2 \mathrm{n}}$ be free parameters. Then for

$$
e^{-\frac{2}{n+1}} \leqq b_{1} \leqq 1
$$

we have

$$
0 \leqq a_{2 n+1} \leqq \frac{1}{n}\left(1-b_{1}^{2 n}\right)
$$

Equality in the case

$$
e^{-\frac{2}{n+1}}<b_{1} \leqq 1
$$

requires that necessarily

$$
a_{n+1}=\ldots=a_{2 n}=0 .
$$


A. I. 435

The conditions $a_{2}=\ldots=a_{2 \mathrm{n}}=0, \quad a_{2 n+1}=\frac{1}{n}\left(1-b_{1}^{2 n}\right) \quad$ can be applied to the extremal conditions of the generalized Nehari inequality [5] to determine the extremal function $f(z)$ completely. It has to satisfy the equation

$$
\frac{f}{\left(1-f^{k-1}\right)^{\frac{2}{k-1}}}=b_{1} \frac{z}{\left(1-z^{k-1}\right)^{\frac{2}{k-1}}}, k=2 n+1 .
$$

The image consists of $2 n$ radial slits with equal lengths and starting at points located at the corners of a regular $2 n$-gon. At the point

$$
b_{1}=e^{-\frac{2}{n+1}}
$$

there is a one-parametric freedom of choice; $a_{n+1}$ can be taken as a parameter. This phenomenon was first observed in the case of $a_{3}$ (see [3], [8]).

\section{4. $a_{2 n+1} ; \delta_{n} \leqq b_{1}<1$}

We shall now drop the assumption (24) and use the fact that for $\delta_{n} \leqq b_{1}<1$ all $a_{v}$ are small. To illustrate the method of estimation to be used, take again $a_{7}$ as an example.

Write (10) in the form

$$
\begin{aligned}
& a_{7}-\frac{1}{3}\left(1-b_{1}^{6}\right) \\
& \leqq \\
\Delta & =\Delta_{1}+\Delta_{2} ; \\
\Delta_{1} & =\frac{1}{2}\left(1-9 b_{1}^{4}\right) a_{2}^{2}+\frac{1}{4}\left(9-25 b_{1}^{2}\right) a_{3}^{2}, \\
\Delta_{2} & =\operatorname{Re}\left\{-9 a_{2} a_{3} t-\frac{19}{12} a_{3}^{3}-\frac{1}{2}\left(3+5 b_{1}^{2}\right) \bar{a}_{2}^{2} a_{3}\right. \\
& \left.+\frac{11}{3} a_{2}^{3} t+\frac{61}{4} a_{2}^{2} a_{3}^{2}-\frac{17}{2} a_{2}^{4} a_{3}+\frac{1}{4}\left(1-b_{1}^{2}\right)^{\prime} a_{2}\right\}^{4} \\
& \left.+\frac{95}{36} a_{2}^{6}\right\} .
\end{aligned}
$$


Denote $\max \left(\left|a_{2}\right|,\left|a_{3}\right|\right)=: M$, and estimate $\Delta_{2}$ by aid of $M$. We find immediately that

$$
\Delta_{2} \leqq M^{2} \cdot \lambda \varepsilon
$$

where

$$
\varepsilon=1-b_{1}
$$

and $\lambda$ is a fixed positive constant which could easily be estimated by aid of (12). There are now two alternatives

1) $\left|a_{2}\right|=M$.
Take $9-25 b_{1}^{2}<0$, which means that $b_{1}>\frac{3}{5}$. Then

$$
\begin{aligned}
& \Delta \leqq\left\lfloor\frac{1}{2}\left(1-9 b_{1}^{4}\right)+\lambda \varepsilon\right]\left|a_{2}\right|^{2} \\
& {[]=-4+(18+\lambda) \varepsilon-27 \varepsilon^{2}+18 \varepsilon^{3}-4,5 \varepsilon^{4} .}
\end{aligned}
$$

We get []$<0$ for $\varepsilon$ small enough. Hence, in this case

$$
a_{7}-\frac{1}{3}\left(1-b_{1}^{6}\right) \leqq 0
$$

for $0<1-b_{1} \leqq \varepsilon_{1} ; 1-\varepsilon_{1} \leqq b_{1}<1$.

2) $\left|a_{3}\right|=M$

Take $1-9 b_{1}^{4}<0, \quad b_{1}>\frac{1}{\sqrt{3}}$.

$$
\Delta \leqq\left[\frac{1}{4}\left(9-25 b_{1}^{2}\right)+\lambda \varepsilon\right]\left|a_{3}\right|^{2} .
$$

As before, we find (33) to hold for $1-\varepsilon_{2} \leqq b_{1}<1$. Denote $\varepsilon_{3}=\max \left(\varepsilon_{1}, \varepsilon_{2}\right)$. The existence of an interval

$$
\delta_{3}=1-\varepsilon_{3} \leqq b_{1}<1
$$

for which (33) holds, is thus established.

The expansions (17), (21) and (23) allow us now to repeat the above procedure in the general case $a_{2 n+1}$. Let us determine the various expressions occuring in inequality (25).

$$
\mathrm{I}=\sum_{i, k=1}^{n} A_{i k} x_{i} x_{k}
$$

Write I as follows:

$$
\mathrm{I}=\sum_{i=1}^{n-1} x_{i} \sum_{k=1}^{n-1} A_{i k} x_{k}+2 \sum_{i=1}^{n-1} A_{i n} x_{i}+A_{n n} .
$$


(17) and (17') give

$$
\left\{\begin{array}{l}
A_{i k}=a_{i+k+1}-\sum_{v=2}^{k+1} C_{v}^{i k} a_{v} a_{i+k+2-v}+\ldots \\
0 \leqq i \leqq k \leqq n, i<n \\
A_{n n}=a_{2 n+1}-\sum_{\nu=2}^{n} v a_{\nu} a_{2 n+2-v}-\frac{n+1}{2} a_{n+1}^{2}+\ldots .
\end{array}\right.
$$

Here the $C_{v}^{i k}$ are fixed constants whose actual value is unimportant. Hence we obtain

$$
\begin{aligned}
\mathrm{I} & =\sum_{i=1}^{n-1} x_{i} \sum_{k=1}^{n-1}\left[a_{i+k+1}-\sum_{v=2}^{k+1} C_{v}^{i k} a_{\nu} a_{i+k+2-v}\right] x_{k} \\
& +2 \sum_{i=1}^{n-1}\left[a_{i+n+1}-\sum_{v=2}^{n+1} C_{v}^{i n} a_{v} a_{i+n+2-v}\right] x_{i} \\
& +a_{2 n+1}-\frac{n+1}{2} a_{n+1}^{2}-\sum_{v=2}^{n} v a_{\nu} a_{2 n+2-v}+\ldots .
\end{aligned}
$$

Consider, in particular, the difference

$$
d=2 \sum_{i=1}^{n-1} a_{i+n+1} x_{i}-\sum_{\nu=2}^{n} v a_{\nu} a_{2 n+2-\nu} .
$$

Denote in the latter sum

$$
v=n+1-i
$$

and obtain for $d$

$$
\begin{aligned}
d & =2 \sum_{i=1}^{n-1} a_{i+n+1} x_{i}-\sum_{i=n-1}^{1}(n+1-i) a_{n+1-i} a_{n+1+i} \\
& =\sum_{i=1}^{n-1}\left[2 x_{i}-(n+1-i) a_{n+1-i}\right] a_{n+1+i} .
\end{aligned}
$$

The parameters $x_{1}, \ldots, x_{n-1}$, which are at our disposal, may be selected to eliminate $d$ :

$$
x_{i}=\frac{n+1-i}{2} a_{n+1-i} ; i=1, \ldots, n-1 .
$$

The above choice implies that

$$
\mathrm{I}=a_{2 n+1}-\frac{n+1}{2} a_{n+1}^{2}+E_{1}
$$

where $E_{1}$ is at least quadratic in $a_{2}, \ldots, a_{n}$ and of third order in all $a_{k}$. 
$\mathrm{II}=\sum_{k=1}^{n} A_{0 k} x_{k}$

It follows from (17) that

$$
\left\{\begin{array}{l}
A_{0 k}=a_{k+1}-\frac{1}{2} \sum_{v=2}^{k} a_{\nu} a_{k+2-v}+\ldots, \\
k=1, \ldots, n
\end{array}\right.
$$

and hence

$$
\mathrm{II}=a_{n+1}+T .
$$

Here $T$ is quadratic in $a_{2}, \ldots, a_{n}$ and contains only these coefficients.

$$
\mathrm{III}=\sum_{i, k=1}^{n} B_{i k} x_{i} \bar{x}_{k}
$$

Again, divide III into the following parts

$$
\mathrm{III}=\sum_{i=1}^{n-1} x_{i} \sum_{k=1}^{n-1} B_{i k} \bar{x}_{k}+2 \operatorname{Re}\left\{\sum_{k=1}^{n-1} B_{n k} \bar{x}_{k}\right\}+B_{n n} .
$$

(21) and (23) give now

$$
\begin{aligned}
& B_{n k}=a_{n-k+1} b_{1}^{2 k}+\ldots ; k=1, \ldots, n-1, \\
& B_{n n}=\frac{1}{n} b_{1}^{2 n}+\sum_{\mu=2}^{n}(n-\mu+1)\left|a_{\mu}\right|^{2} b_{1}^{2(n-\mu+1)}+\ldots,
\end{aligned}
$$

which allows us to write III in the form

$$
\begin{aligned}
\mathrm{III} & =\sum_{i=1}^{n-1}\left|x_{i}\right|^{2} \frac{1}{i} b_{1}^{2 i}+2 \operatorname{Re}\left\{\sum_{k=1}^{n-1} B_{n k} \bar{x}_{k}\right\}+B_{n n}+\ldots \\
& =\sum_{i=1}^{n-1} \frac{1}{i}\left(\frac{n+1-i}{2}\right)^{2} b_{1}^{2 i}+2 \sum_{i=1}^{n-1} \frac{n+1-i}{2}\left|a_{n-i+1}\right|^{2} b_{1}^{2 i} \\
& +\sum_{\mu=2}^{n}(n-\mu+1)\left|a_{\mu}\right|^{2} b_{1}^{2(n-\mu+1)}+\frac{1}{n} b_{1}^{2 n}+\ldots
\end{aligned}
$$

By denoting

$$
i=n+1-\mu
$$

one obtains: 


$$
\begin{aligned}
\mathrm{III} & =\frac{1}{n} b_{1}^{2 n}+\sum_{\mu=2}^{n} \frac{1}{n+1-\mu}\left(\frac{\mu}{2}\right)^{2} b_{1}^{2(n+1-\mu)} \\
& +\sum_{\mu=2}^{n} \mu\left|a_{\mu}\right|^{2} b_{1}^{2(n+1-\mu)}+\sum_{\mu=2}^{n}(n+1-\mu)\left|a_{\mu}\right|^{2} b_{1}^{2(n+1-\mu)}+\ldots
\end{aligned}
$$

which simplifies to

$$
\mathrm{III}=\frac{1}{n} b_{1}^{2 n}+\sum_{\mu=2}^{n} \frac{\left(n+1-\frac{\mu}{2}\right)^{2}}{n+1-\mu}\left|a_{\mu}\right|^{2} b_{1}^{2(n+1-\mu)}+E_{2} .
$$

$E_{2}$ is again of the same nature as $E_{1}$.

Combining I, II and III we arrive at the following form of (25)

$$
\begin{aligned}
& a_{2 n+1}-\frac{1}{n}\left(1-b_{1}^{2 n}\right) \\
& \leqq \frac{n+1}{2} \operatorname{Re}\left\{t^{2}\right\}-\frac{[\operatorname{Re}\{t\}]^{2}}{\log b_{1}^{-1}} \\
& +\sum_{\mu=2}^{n} \frac{1}{n+1-\mu}\left[\left(\frac{\mu}{2}\right)^{2}-\left(n+1-\frac{\mu}{2}\right)^{2} b_{1}^{2(n+1-\mu)}\right]\left|a_{\mu}\right|^{2}+E,
\end{aligned}
$$

where

$$
t=a_{n+1}+T .
$$

Here $E$ is quadratic in $a_{2}, \ldots, a_{n}$, is of third order in all $a_{k}$ and consists only of a finite number of terms.

We are now in the position to arrive again at the conclusion drawn in the case of $a_{7}$. Notice first that

$$
\left(\frac{\mu}{2}\right)^{2}-\left(n+1-\frac{\mu}{2}\right)^{2} b_{1}^{2(n+1-\mu)}<0
$$

if

$$
\left(\frac{\frac{\mu}{2}}{n+1-\frac{\mu}{2}}\right)^{\frac{1}{n+1-\mu}}<b_{1}<1
$$

Since $1 \leqq \frac{\mu}{2} \leqq \frac{n}{2}$, we see that (41) is always valid if

$$
\left(\frac{n}{n+2}\right)^{\frac{2}{n+2}}<b_{1}<1 \text {. }
$$


Further,

$$
\begin{gathered}
\frac{n+1}{2} \operatorname{Re}\left\{t^{2}\right\}-\frac{[\operatorname{Re}\{t\}]^{2}}{\log b_{1}^{-1}} \\
=\left(\frac{n+1}{2}-\frac{1}{\log b_{1}^{-1}}\right)[\operatorname{Re}\{t\}]^{2}-\frac{n+1}{2}[\operatorname{Im}\{t\}]^{2}<0
\end{gathered}
$$

for

$$
e^{-\frac{2}{n+1}}<b_{1}<1
$$

Finally, take into account that

$$
\left\{\begin{array}{l}
\left|a_{v}\right| \leqq k_{\nu} \varepsilon \\
\varepsilon=1-b_{1},
\end{array} \quad(v=2,3, \ldots)\right.
$$

where $k_{v}$ is a constant. This can be deduced for example from Löwner's coefficient representation for bounded functions by a rough estimation [9]. The nature of $E$ allows now to establish that

$$
|E| \leqq M^{2} \lambda_{n} \varepsilon,
$$

where

$$
M=\max \left(\left|a_{2}\right|, \ldots,\left|a_{n}\right|\right)
$$

and $\lambda_{n}$ is a positive constant. Hence we read off from (40) that again the right side of $(40)$ is negative if $0<\varepsilon \leqq \varepsilon_{n}$, i.e.,

$$
\delta_{n}=1-\varepsilon_{n} \leqq b_{1}<1 .
$$

Thus we established the following result which also has been found by SIEWIERSKI by aid of a variational method [6].

Theorem. In the class $S\left(b_{1}\right)$ of bounded univalent functions the sharp inequality

$$
0 \leqq a_{2 n+1} \leqq \frac{1}{n}\left(1-b_{1}^{2 n}\right) \quad(n=1,2, \ldots)
$$

is true at least for some interval

$$
\delta_{n} \leqq b_{1} \leqq 1
$$

where $\delta_{n}$ is a positive number $<1$. 


\section{The coefficient of $\sqrt{f\left(z^{2}\right)}$ and application to general $a_{2 m}$.}

Let us start by considering the expansion

$$
\begin{gathered}
\sqrt{f\left(z^{2}\right)}=b_{1}^{\frac{1}{2}} z\left[1+\sum_{i=1}^{\infty}(-1)^{i-1} \frac{(2 i-2) ! i}{2^{2 i-1}(i !)^{2}}\left(a_{2} z^{2}+\ldots+a_{\mu+1} z^{2 \mu}+\ldots\right)^{i}\right] \\
=b_{1}^{\frac{1}{2}}\left(z+A_{3} z^{3}+\ldots+A_{2 \mu+1} z^{2 \mu+1}+\ldots\right) .
\end{gathered}
$$

We can express the coefficients $A_{2 \mu+1}$ of the new odd function in terms of the coefficients $a_{v}$ of the original function $f(z) \in S\left(b_{1}\right)$. We write

$$
A_{2 \mu+1}=\frac{1}{2} a_{\mu+1}-\frac{1}{4} K_{\mu}+\Delta_{\mu}
$$

and define the terms $K_{\mu}$ and $\Delta_{\mu}$ differently in the cases $\mu=$ even and $\mu=$ odd.

1) If $\mu=2 p, p=1,2,3, \ldots$,

we let

$$
K_{\mu}=K_{2 p}=a_{2} a_{2 p}+a_{3} a_{2 p-1}+\ldots+a_{p} a_{p+2}+\frac{1}{2} a_{p+1}^{2}
$$

and denote by $\Delta_{\mu}=\Delta_{2 p}$ the remainder term which is cubic in $a_{2}, \ldots, a_{2 p}$ and at least quadratic in $a_{2}, \ldots, a_{p}$.

2) If $\mu=2 q+1, q=1,2,3, \ldots$,

we define

$$
K_{\mu}=K_{2 q+1}=a_{2} a_{2 q+1}+a_{3} a_{2 q}+\ldots+a_{q+1} a_{q+2}
$$

$\Delta_{\mu}=\Delta_{2 q+1}$ is quadratic in $a_{2}, \ldots, a_{q+1}$ and cubic in $a_{2}, \ldots, a_{2 q+1}$.

Let us apply the generalised Nehari inequality (5) for

$$
N=2 m-1 \quad(m=2,3, \ldots)
$$

in the case of the function $\sqrt{f\left(z^{2}\right)}$. The highest order coefficient in the inequality is

$$
A_{2 N+1}=A_{4 m-1}=\frac{1}{2} a_{2 m}-\frac{1}{4} K_{2 m-1}+\Delta_{2 m-1} .
$$

It will appear that we can omit the parameters $x_{v}$ with even index:

$$
x_{0}=x_{2}=\ldots=x_{2 m-2}=0 .
$$

This means neglecting the parameter $x_{0}$; hence we actually utilize the original Nehari inequality. Moreover, when maximizing $A_{2 N+1}$ we have to take 


$$
x_{N}=x_{2 m-1}=1 \text {. }
$$

In Section 4 the left side of (25) was decomposed in the expressions I, II and III. We have to rewrite these expressions for the present case as follows:

$$
\mathrm{I}=\sum_{i, k=1}^{N} A_{i k} x_{i} x_{i}
$$

In $\left(35^{\prime}\right)$ we substitute

$$
i=2 r+1, k=2 s+1, v=2 t+1 .
$$

In view of

$$
\left\{\begin{array}{l}
i=1, \ldots, 2 m-3 \\
k=1, \ldots, 2 m-3 \\
v=3, \ldots, 2 m-1
\end{array}\right.
$$

we get for $r, s, t$ :

$$
\left\{\begin{array}{l}
r=0, \ldots, m-2 \\
s=0, \ldots, m-2 \\
t=1, \ldots, m-1
\end{array}\right.
$$

Further, denote

$$
C_{v}^{i k}=C_{2 t+1}^{(2 r+1)(2 s+1)}=D_{t}^{r s}
$$

and find

$$
\begin{aligned}
\mathrm{I} & =\sum_{r=0}^{m-2} x_{2 r+1} \sum_{s=0}^{m-2}\left[A_{2(r+s+1)+1}-\sum_{t=1}^{s} D_{t}^{r s} A_{2 t+1} A_{2(r+s-t+1)+1}\right] x_{2 s+1} \\
& +2 \sum_{r=0}^{m-1}\left[A_{2(r+m)+1}-\sum_{t=1}^{m-1} D_{t}^{r s} A_{2 t+1} A_{2(r+m-t)+1}\right] x_{2 r+1} \\
& +\underline{A_{4 m-1}}-\sum_{t=1}^{m-1}(2 t+1) \underline{A_{2 t+1} A_{2(2 m-t)-1}}+\ldots
\end{aligned}
$$

The underlined coefficients give the following effective contribution to I:

$$
\text { I } \ni \frac{1}{2} a_{2 m}+\sum_{r=0}^{m-2} a_{r+m+1} x_{2 r+1}-\sum_{t=1}^{m-1} \frac{t+1}{2} a_{t+1} a_{2 m-t} .
$$

By denoting

$$
t=m-1-r ; \quad r=m-2, \ldots, 0,
$$

we obtain for this contribution 


$$
\mathrm{I} \ni \frac{1}{2} a_{2 m}+\sum_{r=0}^{m-2}\left[x_{2 r+1}-\frac{m-r}{2} a_{m-r}\right] a_{m+r+1} \text {. }
$$

This suggests the choice

$$
x_{2 r+1}=\frac{m-r}{2} a_{m-r} \quad(r=0, \ldots, m-2)
$$

for the parameters $x_{1}, x_{3}, \ldots, x_{2 m-3}$ at our disposal. Hence (54) is reduced to

$$
\mathrm{I}=\frac{1}{2} a_{2 m}+E_{1}
$$

where $E_{1}$ is quadratic in $a_{2}, \ldots, a_{m}$ and cubic in $a_{2}, \ldots, a_{2 m-1}$.

The number II $=\sum_{k=1}^{N} A_{0 k} x_{k}$ has the coefficient $x_{0}=0$ and therefore no effect in the present case. There remains the combination

$$
\mathrm{III}=\sum_{i, k=1}^{N} B_{i k} x_{i} \bar{x}_{k}
$$

From $\left(38^{\prime}\right)$ we deduce by using (53)

$$
\begin{aligned}
\mathrm{III} & =\sum_{r=0}^{m-2}\left|x_{2 r+1}\right|^{2} \frac{1}{2 r+1} b_{1}^{2 r+1} \\
& +2 \operatorname{Re}\left\{\sum_{s=0}^{m-2} B_{(2 m-1)(2 s+1)} \bar{x}_{2 s+1}\right\}+B_{(2 m-1)(2 m-1)}+\ldots,
\end{aligned}
$$

where

$$
\begin{aligned}
B_{(2 m-1)(2 s+1)} & =A_{2(m-s-1)+1} b_{1}^{2 s+1}+\ldots \\
& =\frac{1}{2} a_{m-s} b_{1}^{2 s+1}+\ldots
\end{aligned}
$$

and

$$
\begin{gathered}
B_{(2 m-1)(2 m-1)}=\frac{1}{2 m-1} b_{1}^{2 m-1}+\sum_{t=1}^{m-1}(2 m-2 t-1)\left|A_{2 t+1}\right|^{2} b_{1}^{2 m-2 t-1}+\ldots \\
=\frac{1}{2 m-1} b_{1}^{2 m-1}+\sum_{\tau=0}^{m-2} \frac{2 m-2 \tau-3}{4}\left|a_{\tau+2}\right|^{2} b_{1}^{2 m-2 \tau-3}+\ldots
\end{gathered}
$$

where

$$
\tau=t-1
$$


This gives

$$
\begin{aligned}
\mathrm{III} & =\sum_{r=0}^{m-2}\left|x_{2 r+1}\right|^{2} \frac{1}{2 r+1} b_{1}^{2 r+1}+\operatorname{Re}\left\{\sum_{s=0}^{m-2} a_{m-s} b_{1}^{2 s+1} \bar{x}_{2 s+1}\right\} \\
& +\frac{1}{2 m-1} b_{1}^{2 m-1}+\sum_{r=0}^{m-2} \frac{2 m-2 \tau-3}{4}\left|a_{r+2}\right|^{2} b_{1}^{2 m-2 r-3}+\ldots .
\end{aligned}
$$

Substituting

$$
s=r, \quad \tau=m-2-r
$$

into (55), and combining similar terms, we finally find

$$
\mathrm{III}=\frac{1}{2 m-1} b_{1}^{2 m-1}+\frac{1}{4} \sum_{r=0}^{m-2} \frac{(m+r+1)^{2}}{2 r+1}\left|a_{m-r}\right|^{2} b_{1}^{2 r+1}+E_{2} .
$$

The omitted terms in $E_{2}$ are of the same nature as in $E_{1}$.

Applying now the formulas (55), (56) and (57) to the inequality (5), we obtain

$$
\mathrm{I}+\mathrm{III} \leqq \frac{1}{2 m-1}+\sum_{r=0}^{m-2} \frac{\left|x_{2 r+1}\right|^{2}}{2 r+1}
$$

which implies that

$$
\begin{gathered}
a_{2 m}-\frac{2}{2 m-1}\left(1-b_{1}^{2 m-1}\right) \\
\leqq \frac{1}{2} \sum_{r=0}^{m-2} \frac{1}{2 r+1}\left[(m-r)^{2}-(m+r+1)^{2} b_{1}^{2 r+1}\right] \mid a_{m-r}{ }^{2}+E \\
(m=2,3, \ldots) .
\end{gathered}
$$

The error term $E$ is quadratic in $a_{2}, \ldots, a_{m}$ and cubic in all $a_{k}$.

The right side of (58) can be estimated as in (40). The estimation is based on the nature of $E$ and the fact that

$$
(m-r)^{2}-(m+r+1)^{2} b_{1}^{2 r+1}<0 \quad(r=0, \ldots, m-2)
$$

for

$$
\left(\frac{m}{m+1}\right)^{2}<b_{1}<1
$$

We end up with

$$
a_{2 m} \leqq \frac{2}{2 m-1}\left(1-b_{1}^{2 m-1}\right)
$$


at least for some interval $\delta_{m} \leqq b_{1} \leqq 1$, where $0<\delta_{m}<1$. Hence we proved:

Theorem. In the class $S\left(b_{1}\right)$ of bounded univalent functions the sharp inequality

$$
0 \leqq a_{k} \leqq \frac{2}{k-1}\left(1-b_{1}^{k-1}\right) \quad(k=2,3, \ldots)
$$

is true at least for some interval

$$
\delta_{k} \leqq b_{1} \leqq 1,0<\delta_{k}<1 .
$$

The result confirms the conjecture by Charzyńsky-Tamm [1], [10]. One extremal function is found by the solution of

$$
\frac{f}{\left(1-f^{k-1}\right)^{\frac{2}{k-1}}}=b_{1} \frac{z}{\left(1-z^{k-1}\right)^{\frac{2}{k-1}}} \quad(k=2,3, \ldots) .
$$

The proof of uniqueness of the extremal function requires a more detailed discussion of the error term. For $a_{2}=\ldots=a_{m}=0$ a result similar to that in Section 3 can be achieved.

$$
\text { 7. } a_{2 m} \text { with } a_{2}=\ldots=a_{m}=0
$$

Let us finally consider the following:

Problem. $a_{2 m}>0$ is to be maximized with the side conditions

$$
a_{2}=\ldots=a_{m}=0 \text {. }
$$

The coefficients $a_{m+1}, \ldots, a_{2 m-1}$ are free parameters of the problem.

In view of (49), the side conditions (63) give for $A_{2 \mu+1}$ the following values:

$$
\left\{\begin{array}{l}
A_{3}=\ldots=A_{2 m-1}=0, \\
A_{2 \mu+1}=\frac{1}{2} a_{\mu+1} \quad(\mu=m, \ldots, 2 m-1) .
\end{array}\right.
$$

The expressions $(54)$ and $\left(56^{\prime}\right)$ simplify considerably in this case:

$$
\begin{aligned}
\mathrm{I} & =\frac{1}{2} \sum_{r=1}^{m-2} x_{2 r+1} \sum_{s=m-1-r}^{m-2} a_{r+s+2} x_{2 s+1} \\
& +\sum_{r=0}^{m-2} a_{r+m+1} x_{2 r+1}+\frac{1}{2} a_{2 m}, \\
\mathrm{III} & =\sum_{r=0}^{m-2}\left|x_{2 r+1}\right|^{2} \frac{1}{2 r+1} b_{1}^{2 r+1}+\frac{1}{2 m-1} b_{1}^{2 m-1} .
\end{aligned}
$$


The above expressions contain no remainder terms because of the side conditions (63).

The inequality (5) assumes now the form

$$
\begin{aligned}
& a_{2 m}-\frac{2}{2 m-1}\left(1-b_{1}^{2 m-1}\right) \\
& \leqq-\operatorname{Re}\left\{\sum_{r=1}^{m-2} x_{2 r+1} \sum_{\mathrm{s}=m-1-r}^{m-2} a_{r+s+2} x_{2 s+1}+2 \sum_{r=0}^{m-2} a_{r+m+1} x_{2 r+1}\right\} \\
& +\sum_{r=0}^{m-2} \frac{2}{2 r+1}\left(1-b_{1}^{2 r+1}\right)\left|x_{2 r+1}\right|^{2} .
\end{aligned}
$$

Exactly as in Section 3, we conclude from this the following

Theorem. Keep $a_{2}=\ldots=a_{m}=0$ and let $a_{m+1}, \ldots, a_{2 m-1}$ be free parameters. Then for $0<b_{1} \leqq 1$

$$
0<a_{2 m} \leqq \frac{1}{2 m-1}\left(1-b_{1}^{2 m-1}\right) \quad(m=1,2, \ldots) .
$$

Equality is only possible if

$$
a_{m+1}=\ldots=a_{2 m-1}=0 .
$$

Stanford University

Stanford, California, U.S.A.

University of Helsinki

Helsinki, Finland. 


\section{References}

[1] Charzyński, Z.: Sur les fonctions univalentes bornées. - Rozprawy Mat. II (1953), 5-6, 25-29.

[2] Garabedian, P. - Schiffer, M.: The local maximum theorem for the coefficients of univalent functions. - Archive for Rational Mechanics and Analysis. Vol. 26, no 1, 1967, pp. 1-32.

[3] Janowski, W.: Le maximum des coefficients $A_{2}$ et $A_{3}$ des fonctions univalentes bornées. - Ann. Polonici Math. 2 (1955).

[4] Nehari, Z.: Some inequalities in the theory of functions. - Trans. Amer. Math. Soc. 75 (1953), pp. $256-286$.

[5] Schiffer, M. - Tammi, O.: On the coefficient problem for bounded univalent functions. - [To appear in Trans. Amer. Math. Soc. (1969)].

[6] - - - - - On the fourth coefficient of bounded univalent functions. - Trans. Amer. Math. Soc. 119 (1965), pp. 67-78.

[7] Siewierski, L.: The local solution of coefficient problem for bounded schlicht functions. - Łodzkie Towarzystwo Naukowe. Soc. Sci. Lodz. III, Nr. 68, 1960.

[8] ТАммг, O.: On bounded univalent functions. - Bulletin de l'Académie Polonaise des Sciences. Série des sci. math., astr. et. phys. - Vol. VII, no 7, 1959.

[9] - - - On the coefficients of the solutions of Löwner's differential equation. Ann. Acad. Sci. Fenn. Ser. A I no 148, 1953.

[10] - - - On the coefficients of bounded schlicht functions. - Comptes rendus du Douziéme Congrés des Mathématiciens Scandinaves tenu á Lund 10-15 août 1953 . 